\title{
Prognostic value of combining c-reactive protein and quick sequential organ failure assessment for adult inpatients: $A$ systematic review
}

\author{
Alexandra Zacharakis \\ Macquarie University \\ Khalia Ackermann \\ Macquarie University \\ Clifford Hughes \\ Macquarie University \\ Vincent Lam \\ Macquarie University \\ Ling Li ( $\square$ ling.li@mq.edu.au ) \\ Macquarie University https://orcid.org/0000-0002-1642-142X
}

\section{Research}

Keywords: qSOFA, PubMed, MEDLINE, EMBASE, Scopus, Web of Science, Science Direct, CINAHL, Open Grey, Grey Literature Report

Posted Date: October 21st, 2021

DOI: https://doi.org/10.21203/rs.3.rs-968626/v1

License: () (i) This work is licensed under a Creative Commons Attribution 4.0 International License. Read Full License 


\section{Abstract}

Objective

To examine the prognostic performance of combining the biomarker c-reactive protein (CRP) with the quick sequential organ failure assessment (qSOFA) bedside tool on mortality prediction in adult inpatients.

Methods

We searched PubMed, MEDLINE, EMBASE, Scopus, Web of Science, Science Direct, CINAHL, Open Grey, Grey Literature Report, and the Clinical Trials registry. Title, abstract, and full text screening were performed by two independent reviewers using pre-determined eligibility criteria. The eligibility criteria were (i) original research, (ii) adult populations, (iii) a comparison between qSOFA and qSOFA combined with CRP, and (iv) set in a hospital environment. The same two reviewers independently extracted data into a pre-designed form and performed a risk of bias assessment using the Quality Assessment tool for Diagnostic Accuracy Studies version 2 (QUADAS-2). Disagreements were settled through discussion and a third reviewer was consulted if necessary. Our primary outcome is mortality.

Results

Three retrospective studies with a total of 1521 patients were included in the review. Adding CRP to qSOFA improved the Area Under the Receiver Operating Characteristic Curve (AUROC) value in all three studies by $3-10 \%$. In the two studies with available data, the addition of CRP improved the sensitivity of qSOFA for mortality risk stratification by $43 \%$ and $71 \%$, while decreasing the specificity by $12 \%$ and $7 \%$ respectively. The cut-off values of CRP ranged from 60 to $128.8 \mathrm{mg} / \mathrm{L}$ across the three studies. A meta-analysis was not performed due to the heterogeneity across studies and the small sample size.

Conclusions

This comprehensive review provides initial evidence that combining CRP with qSOFA could improve the prognostic performance of qSOFA alone in identifying patients at risk of dying in hospital. The combined tool demonstrates potential to improve patient outcomes, especially in low resource settings. The review also reveals research gaps in this area.

Registration

PROSPERO registration No. CRD42020190973

\section{Introduction}

Sepsis is a leading cause of mortality, with global estimates attributing 11 million deaths to sepsis in 2017 alone.[1] The most recent definition of sepsis, published in 2016, is "life threatening organ dysfunction caused by a dysregulated host response to infection".[2] Patients who do not receive prompt identification and management rapidly deteriorate, contributing to the high mortality rate.[2,3] Thus, identifying septic patients and initiating evidence based bundle care is paramount to improving sepsis outcomes.[1] However, despite numerous efforts to develop effective diagnostic tools, rapid sepsis identification remains a global challenge.[4]

In 2016, the quick sepsis-related organ failure assessment (qSOFA) was introduced as a simple bedside scoring system aimed to identify patients at higher risk of poor sepsis related outcomes.[2] qSOFA implements 3 clinical components: altered mentation determined via a Glasgow Coma Scale (GCS) less than 15, a respiratory rate of 22 breaths per minute or greater, and a systolic blood pressure of $100 \mathrm{mmHg}$ or less.[2] A score of 2 or more indicates a patient is more likely to experience poor outcomes typical of sepsis, such as ICU admission or death.[2] Since its integration into clinical care, the poor sensitivity of qSOFA for mortality prediction and risk stratification of sepsis has raised concerns. $[5,6]$

C-reactive protein (CRP) has been shown to be highly sensitive for identifying sepsis.[7, 8] CRP is an acute phase reactant released by the liver in the early stages of inflammation and infection.[9-11] Circulating levels of CRP are impacted by a myriad of factors, including; age, sex, smoking status, weight, blood lipid levels, and blood pressure.[9] Critically, CRP is one of the first biomarkers to rise in response to infection.[9] Studies have shown CRP blood levels to rise six hours after stimulus, peaking at 48 hours, with a halflife of approximately 19 hours.[12] In addition, CRP is accessible and inexpensive.[10,13] Studies in low resource settings, such as 
Nepal[7] and rural Congo[14] have shown the merit of CRP in assisting clinicians to determine the severity of bacterial infections. Studies have also shown CRP point of care testing to be accessible and cost-effective in guiding antibiotic use in febrile patients. [15-18] Therefore, including CRP in qSOFA may improve qSOFA's sensitivity and ability to accurately stratify a patient's mortality risk in both high and low resource settings.

The current evidence evaluating the performance of qSOFA combined with CRP is limited. Preliminary searches suggest a combined approach of qSOFA and CRP yields improved performance when compared to qSOFA alone.[19] However, study populations have been small and constrained to specific patient conditions.[19] Moreover, there has been no systematic approach appraising the literature, and therefore, a systematic review analysing the benefit of qSOFA combined with CRP is warranted. This paper aimed to systematically evaluate the prognostic value of CRP combined with qSOFA, compared to qSOFA alone, in predicting mortality among adult hospital patients.

\section{Methods}

\section{Protocol registration}

This study complied with the recommendations for the conduct and reporting of systematic reviews and meta-analyses, set forth by the Preferred Reporting Items for Systematic Reviews and Meta-Analyses for Diagnostic Test Accuracy (PRISMA-DTA) statement.[20] The study protocol was developed and registered with PROSPERO (Registration No. CRD42020190973).

\section{Literature search and study selection}

A comprehensive electronic search of eight databases (Figure 1), including PubMed, Embase, MEDLINE, Scopus, CINAHL, Web of Science, Science Direct, Clinical Trials Registry, Open Grey and the Grey Literature Report was performed (see Appendix for the search strategies). Reference lists were also hand searched for any additional relevant studies.

No restrictions were placed on publication date, allowing complete coverage of included databases. Publications were restricted to original research. The search was completed on the 8th of August 2020. Following deduplication of the search results, two review authors (AZ, KA) independently performed title, abstract and keyword screening, whereby pre-decided eligibility criteria (see next section) were applied to all results.

References that were relevant to the topic after title, abstract and key word screening had full text articles retrieved. Subsequently, they underwent full text screening and the same, pre-decided, eligibility criteria were applied. Any disagreements during the search and screening process were settled via discussion or consultation with a third team member (LL) to reach consensus.

\section{Eligibility criteria}

Studies had to meet all of the inclusion criteria and none of the exclusion criteria to be included in this review. The inclusion criteria were: (i) study population aged $\geq 18$ years or adult patients as defined in the papers, (ii) a comparison between qSOFA (reference test) to qSOFA combined with CRP (index test), (iii) study set in a hospital environment, (iv) original research performed regardless of study design, and ( $v$ ) published in English or with a readily available English translation. We accepted any combination of CRP and qSOFA used by the study authors. We defined hospital setting as either emergency department, intensive care unit or ward environment. We only accepted papers in English or with English translations readily available due to time and resource constraints.

The exclusion criteria were: (i) study population younger than 18 years old and (ii) papers based on opinions or expert views. The study population was confined to patients 18 years or older, as paediatric and neonatal populations have unique responses to sepsis at the cellular, organ, and whole organism level.[21]

\section{Outcomes}

Our primary outcome is the accuracy of patient mortality risk stratification.

\section{Data extraction}

All relevant study characteristics and design information were extracted into a pre-designed excel template, which included: country of study, study design, sample size, clinical context of study, setting of study, and study population demographics (sex, age, 
comorbidities). Similarly, all relevant outcome information were extracted, where possible, into a pre-designed Excel template, which included: overall mortality rates, and performance outcomes for qSOFA and qSOFA combined with CRP (i.e. sensitivity, specificity, area under receiving operating characteristics curve (AUROC), negative predictive value (NPV) and positive predictive value (PPV)). Data extraction was performed independently by two reviewers (AZ, KA), with any disagreements being settled through discussion or by consultation with a third team member (LL). For any missing data, the relevant author was contacted via email, and in one case this was successful.[22]

\section{Quality assessment}

Two authors (AZ, KA) independently assessed the quality of each full text article via the Quality Assessment of Diagnostic Accuracy Studies 2 (QUADAS-2) tool, which is recommended by the Cochrane Collaboration for the quality assessment of diagnostic studies. [23] Any disagreements were resolved by discussion and consultation with a third reviewer (LL). The QUADAS-2 tool consists of four domains, including: patient selection, index test, reference standard, and flow and timing.[23] It aims to assist systematic review authors to rate the risk of bias and applicability in studies investigating diagnostic test accuracy. Each domain has a grade of low, high or unclear for its risk of bias and its concerns regarding applicability.

\section{Data synthesis}

Data synthesis was limited to narrative synthesis and summary statistics. Data regarding study characteristics (country of study, study design, sample size, clinical context of study, setting of study) and participant characteristics (sex, age, comorbidities) were synthesised into tables, which enabled comparison across the studies. For papers with available data, $2 \times 2$ tables comparing the number of patients with qSOFA $\geq 2$, qSOFA $<2$, qSOFA+CRP $\geq 2$, and qSOFA+CRP $<2$ in survivors and non-survivors was designed. The sensitivity, specificity, negative predictive value and positive predictive value of the test for mortality risk prediction were calculated if not provided in the original papers. For papers with data not available to complete the table, Yu et al[24] and Kim et al[22], study authors were contacted via email. Kim et al[22] provided the requested raw data for us. To create the appropriate $2 \times 2$ tables from this raw data, we defined qSOFA combined with CRP as a four-point scoring system with one point attributed for: altered mental status, increased respiratory rate, decreased systolic blood pressure, and elevated CRP.

\section{Results}

\section{Search results}

Database searching retrieved 179 studies. After duplicates were removed, 83 articles underwent title, abstract and keyword screening (Figure 1). The full texts of 17 studies were screened, and subsequently, three papers met the inclusion criteria and were included in the final analysis.[19, 22, 24] Reasons for exclusion at this stage were recorded (Figure 1).

\section{Characteristics of studies}

The characteristics of included studies and the characteristics of survivors and non-survivors are presented in Tables 1 and 2 respectively. Evaluating qSOFA combined with CRP was the primary aim for only one study.[19] The remaining two studies included qSOFA combined with CRP as a secondary aim. $[22,24]$ All studies were retrospective cohort studies with two performed at single centres, and one conducted across three hospital sites (Table 1).[19, 22, 24] Studies varied in their CRP cut off value. Yu et al[24] had the lowest CRP cut off value ( $>60 \mathrm{mg} / \mathrm{L})$ and $\mathrm{Kim}$ et al[22] had the greatest CRP cut off value $(>128.8 \mathrm{mg} / \mathrm{L})$. All studies involved patients presenting to emergency departments with or without subsequent admission. Differences between survivors and nonsurvivors were apparent in age, presence of malignancy and presence of chronic renal failure (Table 2). However, all three studies had similar age and comorbidity distributions in their study populations, with diabetes and malignancy being present across all three studies.[19, 22, 24] 
Table 1

Characteristics of included studies

\begin{tabular}{|c|c|c|c|c|c|c|c|c|}
\hline Study & Country & Study design & $\begin{array}{l}\text { Number of } \\
\text { participants }\end{array}$ & $\begin{array}{l}\text { Clinical } \\
\text { context }\end{array}$ & Setting & $\begin{array}{l}\text { CRP cut off } \\
\text { for } \\
\text { qSOFA+CRP } \\
\text { score } \\
\text { (mg/L) } \\
\text { (point) }\end{array}$ & $\begin{array}{l}\text { Cut off } \\
\text { score for } \\
\text { qSOFA+CRP }\end{array}$ & $\begin{array}{l}\text { Patient } \\
\text { outcome }\end{array}$ \\
\hline $\begin{array}{l}\text { Dimitrov } \\
\text { et al } \\
(2019)[19]\end{array}$ & Bulgaria & $\begin{array}{l}\text { Retrospective } \\
\text { study }\end{array}$ & 78 & $\begin{array}{l}\text { Patients } \\
\text { referred } \\
\text { from ED } \\
\text { who } \\
\text { underwent } \\
\text { surgery for } \\
\text { complicated } \\
\text { abdominal } \\
\text { infections }\end{array}$ & $\begin{array}{l}\text { Department } \\
\text { of surgical } \\
\text { diseases - } \\
\text { in hospital }\end{array}$ & $\begin{array}{l}>100(1 \\
\text { point) }\end{array}$ & $\geq 2$ & $\begin{array}{l}\text { In- } \\
\text { hospital } \\
\text { mortality }\end{array}$ \\
\hline $\begin{array}{l}\text { Yu et al } \\
\text { (2019)[24] }\end{array}$ & $\begin{array}{l}\text { China } \\
\text { and } \\
\text { Taiwan }\end{array}$ & $\begin{array}{l}\text { Retrospective } \\
\text { multi-centre } \\
\text { cohort study }\end{array}$ & 1318 & $\begin{array}{l}\text { Patients } \\
\text { presenting } \\
\text { to ED or } \\
\text { who were } \\
\text { admitted to } \\
\text { hospital } \\
\text { with } \\
\text { symptoms } \\
\text { that } \\
\text { indicated } \\
\text { systemic } \\
\text { infection }\end{array}$ & 3 hospitals & $\begin{array}{l}60-120 \text { (1 } \\
\text { point); } \\
>120(2 \\
\text { points) }\end{array}$ & $\geq 2$ & $\begin{array}{l}\text { In- } \\
\text { hospital } \\
\text { mortality }\end{array}$ \\
\hline $\begin{array}{l}\text { Kim et al } \\
(2017)[22]\end{array}$ & Korea & $\begin{array}{l}\text { Retrospective } \\
\text { chart review }\end{array}$ & 125 & $\begin{array}{l}\text { Patients } \\
\text { admitted to } \\
\text { ED with } \\
\text { discharge } \\
\text { diagnosis } \\
\text { of } \\
\text { community } \\
\text { acquired } \\
\text { pneumonia }\end{array}$ & Hospital & $\begin{array}{l}>128.8(1 \\
\text { point) }\end{array}$ & $\geq 2$ & $\begin{array}{l}\text { 28-day } \\
\text { mortality }\end{array}$ \\
\hline
\end{tabular}


Table 2

Survivor vs non-survivor patient characteristics

\begin{tabular}{|c|c|c|c|c|}
\hline & & Dimitrov et al (2019) [19] & Yu et al (2019) [24] & Kim et al (2017) [22] \\
\hline \multirow[t]{2}{*}{ Age, years; Mean \pm SD } & Survivors & $54.21 \pm 18.29$ & 62 (47-74) (median, IQR) & $67.2 \pm 18.0$ \\
\hline & Non-survivors & $73.25 \pm 12.18$ & $71(55-81)$ (median, IQR) & $76.4 \pm 9.5$ \\
\hline \multirow[t]{2}{*}{ Male; n (\%) } & Survivors & $33(76.7)$ & 708 (62.2) & $70(62.5)$ \\
\hline & Non-survivors & $10(50)$ & $118(66.3)$ & $8(61.5)$ \\
\hline \multirow[t]{2}{*}{ Diabetes mellitus; n (\%) } & Survivors & $6(10.3 \%)$ & $253(22.2)$ & $30(26.8)$ \\
\hline & Non-survivors & $3(15.0)$ & $118(66.3)$ & 8 (61.5) \\
\hline \multirow[t]{2}{*}{ Hypertension; n (\%) } & Survivors & $20(30.4)$ & NR & $46(41.1)$ \\
\hline & Non-survivors & $10(50.0)$ & NR & $9(69.2)$ \\
\hline \multirow[t]{2}{*}{ Malignancy; n (\%) } & Survivors & $7(12.1)$ & $76(6.7)$ & $25(22.3)$ \\
\hline & Non-survivors & $8(40.0)$ & $26(14.6)$ & $2(15.4)$ \\
\hline \multirow[t]{2}{*}{ Chronic renal failure; n (\%) } & Survivors & $1(1.7)$ & NR & $11(9.8)$ \\
\hline & Non-survivors & $5(25.0)$ & NR & $4(30.8)$ \\
\hline \multirow[t]{2}{*}{ Chronic liver disease; n (\%) } & Survivors & NR & $84(6.4)$ & $3(2.7)$ \\
\hline & Non-survivors & NR & $67(5.9)$ & 0 \\
\hline
\end{tabular}

\section{AUROC values}

The AUROC values were able to be extracted from each paper without any data synthesis and are illustrated in Table 3.[19, 22, 24] Across all studies there is a universal increase in AUROC values with the addition of CRP to qSOFA.[19, 22, 24]

Table 3

Combined sensitivity, specificity, PPV, NPV and AUROC for qSOFA and qSOFA+CRP

\begin{tabular}{|lllllll|}
\hline Study & Test & AUROC $(\mathbf{9 5 \%}$ Cl) & Sensitivity & Specificity & PPV & NPV \\
\hline Dimitrov et al (2019)[19] & qSOFA & $0.746(0.603-0.889)$ & 0.350 & 0.983 & 0.875 & 0.814 \\
\cline { 2 - 7 } & qSOFA+CRP & $0.818(0.704-0.932)$ & 0.600 & 0.914 & 0.706 & 0.869 \\
\hline Yu et al (2019)[24] & qSOFA & $0.670(0.620-0.710)$ & NR & NR & NR & NR \\
\cline { 2 - 7 } & qSOFA+CRP & $0.690(0.640-0.730)$ & NR & NR & NR & NR \\
\hline Kim et al (2017)[22] & qSOFA & $0.810(0.730-0.87)$ & $0.538^{*}$ & $0.892^{*}$ & $0.368^{*}$ & $0.943^{*}$ \\
\cline { 2 - 7 } & qSOFA+CRP & $0.870(0.790-0.920)$ & $0.769 *$ & $0.785^{*}$ & $0.294^{*}$ & $0.967^{*}$ \\
\hline NR = not reported; * Supplemental data supplied by study authors via email. & & & \\
\hline
\end{tabular}

\section{Sensitivity and specificity: mortality prediction}

Using $2 \times 2$ tables (see Appendix), it was possible to determine the sensitivity and specificity for two studies, Dimitrov et al[19] and Kim et al[22], with the results shown in Table 3. The sensitivity of qSOFA was poor across both studies at 0.350 and 0.538 , respectively. However, with the addition of CRP scoring, the sensitivity increased in both studies to 0.600 and $0.769 .[19,22]$ This indicates addition of CRP to qSOFA increased sensitivity by $71.43 \%$ and $42.94 \% .[19,22]$ Conversely, the addition of CRP scoring decreased the specificity compared to qSOFA alone. Using qSOFA alone the specificity was 0.983 and 0.892 in Dimitrov et al[19] and Kim et al[22] respectively. When using qSOFA combined with CRP the specificity decreased to 0.914 and $0.785 .[19,22]$ This indicates the addition 
of CRP to qSOFA decreased the specificity by $7.02 \%$ and $12.00 \% .[19,22]$ Ultimately, increasing the sensitivity of qSOFA through the addition of a CRP score, resulted in a subsequent decrease in specificity.

\section{Negative predictive value and positive predictive value: mortality prediction}

Similarly, the 2x2 tables were used to calculate the positive predictive value (PPV) and negative predictive value (NPV) for both qSOFA alone and qSOFA combined with CRP, with results illustrated in Table 3. The PPV for qSOFA was 0.875 and 0.368 in studies conducted by Dimitrov et al[19] and Kim et al[22], respectively. The PPV for qSOFA combined with CRP was 0.706 and 0.294.[19, 22] This indicates the addition of CRP to qSOFA decreased PPV by $19.31 \%$ and $20.11 \%$.[19, 22] The NPV for qSOFA was 0.814 and 0.943 across the two studies, Dimitrov et al[19] and Kim et al[22]. Conversely, the NPV for qSOFA combined with CRP was 0.869 and 0.967, respectively.[19, 22] This indicates addition of CRP to qSOFA resulted in an increase in NPV by $6.76 \%$ and $2.55 \%$.[19, 22]

\section{Risk of Bias results}

Upon assessment of QUADAS-2, there were no concerns regarding risk of bias or applicability for any paper, with only one unclear point in regards to the reference standard used for Kim et al[22] (Figure 2).[23]

\section{Discussion}

Sepsis remains a global challenge, with an estimated 50 million people suffering from sepsis worldwide in 2017 alone.[1] This comprehensive review presents evidence demonstrating that a combined qSOFA and CRP score improved the prognostic performance of qSOFA alone for mortality prediction, which has previously been shown to be suboptimal.[25] In all three included studies, the addition of a CRP score resulted in improved AUROC values for mortality prediction compared to qSOFA alone.[19, 22, 24] Furthermore, in the two studies with available data, adding CRP to the qSOFA tool increased the sensitivity and NPV compared to qSOFA. $[19,22]$ In contrast, the addition of CRP to qSOFA resulted in a reduced specificity and PPV for mortality prediction.[19, 22] This is expected as sensitivity and specificity are inversely related, and therefore an increase in a test's sensitivity will cause a decrease in its specificity.[26] As sepsis is a highly-fatal and time-critical condition having a higher sensitivity results in a greater likelihood of identifying septic patients earlier in the disease course, and consequently, offers improved chances of better outcomes. [27] Therefore, it is acceptable to have a lower specificity in favour of a higher sensitivity for the early identification of sepsis.

Our findings present an opportunity for improved early detection of patients with a higher risk of poor outcomes from sepsis, especially in the emergency setting. qSOFA combined with CRP is most suited to fast paced, high patient flow-through settings where the emphasis is on rapid and sensitive risk stratification assessments. Furthermore, as CRP is one of the earliest biomarkers to rise in response to infection, it is well placed to be implemented in a clinical environment where patients are initially presenting.[12] Use of qSOFA combined with CRP is less beneficial in ward and intensive care environments as there is more time, patient history and resources to inform clinical decision making. Moreover, a CRP value is easily obtainable via venous collection and is a relatively inexpensive test with results obtainable quickly.[28] Due to its early rise in infection, ease of access, cost-effectiveness, and broad applicability, CRP is an ideal biomarker to combine with qSOFA for use in the emergency setting.

The patient populations of the included papers presented with a spectrum of conditions, including complicated abdominal infections, community acquired pneumonia, and symptoms suggesting systemic infection.[19, 22, 24] This reflects the variation in sepsis presentations and impacted patient populations. In addition, the patient populations in these studies had comorbidities widely present in the community including diabetes, hypertension, malignancy, and chronic renal and liver disease. Therefore, this review presents initial evidence showing qSOFA combined with CRP is useful in a spectrum of patient populations, including those with common comorbidities.

Implications of this novel research are particularly prominent for developing countries. Research has shown the need for a test to facilitate early septic patient detection in low resource settings, as they carry the majority of sepsis incidence and mortality globally. $[1,29]$ We defined low resource setting according to a recent systematic review that thematically approaches the term. [30] We applied the themes: financial pressure, geographical and environmental factors, to define low resource settings.[30] Importantly, CRP measurement can be achieved through inexpensive and rapid PoC testing, which can be performed at the bedside.[31, 32] Similarly, the qSOFA score components are simple and inexpensive bedside measurements.[33] Thus, the combined qSOFA and CRP score offers a low-cost prognostic and risk stratification tool that incorporates clinical and biochemical scores to improve the timely identification of patients with a high risk of sepsis-related poor outcomes.[15, 16] Earlier recognition of such patients can improve time to antibiotics initiation, and hence could improve mortality and morbidity rates of sepsis.[16, 17] Therefore, the combined qSOFA 
and CRP score shows promise as a tool for early sepsis identification in low resource settings and is a valuable area for further research.

The studies included in this systematic review implemented a wide spectrum of CRP cut-off values, ranging from $60 \mathrm{mg} / \mathrm{L}$ to $128.8 \mathrm{mg} / \mathrm{L} .[19,22,24]$ One study included two cut-off values for the combined qSOFA and CRP score[24] and all three studies used similar CRP thresholds of $100-128.8 \mathrm{mg} / \mathrm{L}$. The CRP threshold chosen will influence the risk stratification of patients. Some previous studies have indicated that the optimal CRP cut-off value for sepsis identification in adults is 61 to $84 \mathrm{mg} / \mathrm{L}$.[7, 12] However, the optimal CRP threshold could be influenced heavily by age.[34-36] In addition, studies included in this review involved patients with community acquired pneumonia, complicated abdominal infections, and a range of comorbidities.[19, 22, 24] As sepsis impacts a diverse range of patients with varying and often complex medical histories, future research should investigate the role of age, comorbidities, and common concurrent conditions on the reliability of CRP cut-off values for the diagnosis of sepsis. Furthermore, future studies investigating CRP in combination with qSOFA should endeavour to use an evidence-based and consistent threshold as a cut off, to improve validity and reliability between studies.

Other biomarkers, such as procalcitonin (PCT), can be added to the combination of CRP and qSOFA to further increase the sensitivity or specificity of the score. Recent studies have shown merit of using a combination of PCT and CRP in diagnosing sepsis in both neonatal and adult populations.[37, 38] Further research should investigate the role of other biomarkers in combination with CRP and qSOFA.

The quality of evidence presented in this review is of an acceptable standard, evaluated using the QUADAS-2 tool.[23] All studies were recently published, using the most up to date definition of sepsis, sepsis-3.[2] A key methodological limitation across all studies is that it is unclear if the reference standard results, qSOFA, were interpreted without knowledge of results of the index test, qSOFA combined with CRP. However, as all studies were retrospective analyses, there is a low possibility for the introduction of bias, as the data has already been collated. Overall, as evidenced in the QUADAS-2 risk of bias assessment, the three studies included in this review were of low risk of bias with low concerns regarding applicability of studies.

A strength of this review is the systematic and comprehensive literature search with a robust search strategy that included multiple databases, grey literature and hand searching. Thereby, obtaining the best likelihood that all relevant papers were included. A limitation of this review is it was restricted to papers written in English or those with an English translation readily available. This was done due to time and financial constraints. Additionally, a valid meta-analysis could not be performed for two primary reasons. Firstly, there was not enough data to calculate standardised effect sizes. Study authors were contacted via email to seek additional information, however, only one replied and supplied additional data.[22] Secondly, the limited number of studies included $(n=3)$ displayed substantial heterogeneity in the clinical setting, CRP cut-off values and included patient populations.

\section{Conclusion}

Sepsis remains a global challenge, with best outcomes yielded from rapid identification and risk stratification of septic patients. Our comprehensive review demonstrates that inclusion of CRP to qSOFA confers improved prognostic performance of identifying patients at risk of poor outcomes, compared to qSOFA alone. Critically, the addition of CRP to qSOFA improved the sensitivity of qSOFA. This review yields new evidence for an improved tool to identify the septic patient in both high and low resource settings. We have shown that the combination of qSOFA and CRP could be beneficial for an ED setting as it is a rapid PoC test and CRP is one of the earliest biomarkers to rise in sepsis patients. Further research into the combined CRP and qSOFA score is encouraged to confirm these promising preliminary findings, with a focus to investigate the performance of CRP combined with qSOFA in larger patient cohorts. This would contribute to determining the optimal cut-off level for CRP when combined with qSOFA, and the role of other biomarkers in combination with qSOFA.

\section{Declarations}

\section{Ethics approval and consent to participate}

Not applicable. Ethics approval is not required as this is a systematic review and used data already published.

\section{Consent for publication}

Page $8 / 12$ 
Not applicable

\section{Availability of data and materials}

The datasets used and/or analysed during the current study are available from the corresponding author on reasonable request.

\section{Competing interests}

The authors declare that they have no competing interests.

\section{Funding}

This research received no specific grant from any funding agency in the public, commercial or not-for-profit sectors.

\section{Authors' contributions}

AZ planned the study objectives, designed, and executed the search across all databases, performed title, abstract, keyword, and full text screening, extracted data, performed risk of bias analysis, and drafted the final manuscript. KA performed title, abstract, keyword, and full text screening, extracted data, performed risk of bias analysis, and reviewed the manuscript. CH \& VL assisted with project design and manuscript review. LL was responsible for overall project, study design, being consulted on issues during screening, extraction, and risk of bias, and manuscript review. All authors read and approved the final manuscript.

\section{ACKNOWLEDGEMENTS}

The authors would like to thank our librarian, Ms Mary Simons, for her expertise in refining the search strategy and translating it for other databases.

\section{References}

1. Rudd KE, Johnson SC, Agesa KM, et al. Global, regional, and national sepsis incidence and mortality, 1990-2017: analysis for the Global Burden of Disease Study. Lancet. 2020;395(10219):200-11.

2. Singer M, Deutschman CS, Seymour CW, et al. The Third International Consensus Definitions for Sepsis and Septic Shock (Sepsis-3). Jama. 2016;315(8):801-10. 26903338.

3. Vincent J-L. The Clinical Challenge of Sepsis Identification and Monitoring. PLoS Med. 2016;13(5):e1002022-2. 27187803.

4. Reyna MA, Josef CS, Jeter R, et al. Early Prediction of Sepsis From Clinical Data: The PhysioNet/Computing in Cardiology Challenge 2019. Crit Care Med. 2020;48(2):210-7. 31939789.

5. Dykes LA, Heintz SJ, Heintz BH, Livorsi DJ, Egge JA, Lund BC. Contrasting qSOFA and SIRS Criteria for Early Sepsis Identification in a Veteran Population. Fed Pract. 2019;36(Suppl 2):21-4. 30983857.

6. Usman OA, Usman AA, Ward MA. Comparison of SIRS, qSOFA, and NEWS for the early identification of sepsis in the Emergency Department. Am J Emerg Med. 2019;37(8):1490-7. 30470600.

7. Pradhan S, Ghimire A, Bhattarai B, et al. The role of C-reactive protein as a diagnostic predictor of sepsis in a multidisciplinary Intensive Care Unit of a tertiary care center in Nepal. Indian J Crit Care Med. 2016;20(7):417-20. 27555697.

8. Póvoa P, Coelho L, Almeida E, et al. C-reactive protein as a marker of infection in critically ill patients. Clin Microbiol Infect. 2005;11(2):101-8.

9. Sproston NR, Ashworth JJ. Role of C-Reactive Protein at Sites of Inflammation and Infection. Front Immunol. 2018;9:754-4. 29706967.

10. Sugden K, Danese A, Shalev I, Williams BS, Caspi A. Blood Substrate Collection and Handling Procedures under Pseudo-Field Conditions: Evaluation of Suitability for Inflammatory Biomarker Measurement. Biodemography Soc Biol. 2015;61(3):273-84. 26652682.

11. Faix JD. Biomarkers of sepsis. Crit Rev Clin Lab Sci. 2013;50(1):23-36. 23480440.

12. Nelson GE, Mave V, Gupta A. Biomarkers for sepsis: a review with special attention to India. Biomed Res Int. 2014;2014:264351. 24772418. 
13. Kip MMA, van Oers JA, Shajiei A, et al. Cost-effectiveness of procalcitonin testing to guide antibiotic treatment duration in critically ill patients: results from a randomised controlled multicentre trial in the Netherlands. Crit Care. 2018;22(1):293. 30424796.

14. Bottieau E, Mukendi D, Kalo J-RL, et al. Potential usefulness of C-reactive protein and procalcitonin determination in patients admitted for neurological disorders in rural Democratic Republic of Congo. Scientific Reports. 2019;9(1):15505.

15. Escadafal C, Incardona S, Fernandez-Carballo BL, Dittrich S. The good and the bad: using C reactive protein to distinguish bacterial from non-bacterial infection among febrile patients in low-resource settings. BMJ Global Health. 2020;5(5):e002396.

16. Althaus T, Greer RC, Swe MMM, et al. Effect of point-of-care C-reactive protein testing on antibiotic prescription in febrile patients attending primary care in Thailand and Myanmar: an open-label, randomised, controlled trial. Lancet Glob Health. 2019;7(1):e119-31. 30554748.

17. Do NT, Ta NT, Tran NT, et al. Point-of-care C-reactive protein testing to reduce inappropriate use of antibiotics for non-severe acute respiratory infections in Vietnamese primary health care: a randomised controlled trial. Lancet Glob Health. 2016;4(9):e633-41. 27495137.

18. Oppong R, Jit M, Smith RD, et al. Cost-effectiveness of point-of-care C-reactive protein testing to inform antibiotic prescribing decisions. British Journal of General Practice. 2013;63(612):e465.

19. Dimitrov E, Minkov G, Enchev E, Halacheva K, Yovtchev Y. A combination of C-reactive protein and quick sequential organ failure assessment (qSOFA) score has better prognostic accuracy than qSOFA alone in patients with complicated intra-abdominal infections. Acta Chir Belg. 2019:1-5. 31307292.

20. Page MJ, Moher D, Bossuyt PM, et al. PRISMA 2020 explanation and elaboration: updated guidance and exemplars for reporting systematic reviews. BMJ. 2021;372:n160.

21. Wheeler DS, Wong HR, Zingarelli B. Pediatric Sepsis - Part I: "Children are not small adults!". Open Inflamm J. 2011;4:4-15. 23723956.

22. Kim MW, Lim JY, Oh SH. Mortality prediction using serum biomarkers and various clinical risk scales in community-acquired pneumonia. Scandinavian Journal of Clinical Laboratory Investigation. 2017;77(7):486-92.

23. Whiting PF, Rutjes AW, Westwood ME, et al. QUADAS-2: a revised tool for the quality assessment of diagnostic accuracy studies. Ann Intern Med. 2011;155(8):529-36. 22007046.

24. Yu H, Nie L, Liu A, et al. Combining procalcitonin with the qSOFA and sepsis mortality prediction. Medicine (Baltimore). 2019;98(23). WOS:000480721300069.

25. Fernando SM, Tran A, Taljaard M, et al. Prognostic Accuracy of the Quick Sequential Organ Failure Assessment for Mortality in Patients With Suspected Infection: A Systematic Review and Meta-analysis. Ann Intern Med. 2018;168(4):266-75. 29404582.

26. Parikh R, Mathai A, Parikh S, Chandra Sekhar G, Thomas R. Understanding and using sensitivity, specificity and predictive values. Indian J Ophthalmol. 2008;56(1):45-50. 18158403.

27. Liu VX, Fielding-Singh V, Greene JD, et al. The Timing of Early Antibiotics and Hospital Mortality in Sepsis. Am J Respir Crit Care Med. 2017;196(7):856-63. 28345952.

28. Ryu J-A, Yang JH, Lee D, et al. Clinical Usefulness of Procalcitonin and C-Reactive Protein as Outcome Predictors in Critically III Patients with Severe Sepsis and Septic Shock. PLoS One. 2015;10(9):e0138150-0. 26367532.

29. Liu Y, Hou JH, Li Q, Chen KJ, Wang SN, Wang JM. Biomarkers for diagnosis of sepsis in patients with systemic inflammatory response syndrome: a systematic review and meta-analysis. Springerplus. 2016;5(1):2091. 28028489.

30. van Zyl C, Badenhorst M, Hanekom S, Heine M. Unravelling 'low-resource settings': a systematic scoping review with qualitative content analysis. BMJ Global Health. 2021;6(6):e005190.

31. Yager P, Domingo GJ, Gerdes J. Point-of-care diagnostics for global health. Annu Rev Biomed Eng. 2008;10:107-44. 18358075.

32. Oeschger T, McCloskey D, Kopparthy V, Singh A, Erickson D. Point of care technologies for sepsis diagnosis and treatment. Lab Chip. 2019;19(5):728-37. 30724931.

33. Andaluz D, Ferrer R. SIRS, qSOFA, and organ failure for assessing sepsis at the emergency department. J Thorac Dis. 2017;9(6):1459-62. 28740658.

34. Liu A, Bui T, Van Nguyen H, Ong B, Shen Q, Kamalasena D. Serum C-reactive protein as a biomarker for early detection of bacterial infection in the older patient. Age Ageing. 2010;39(5):559-65. 20573779.

Page 10/12 
35. Zhang H, Wang X, Zhang Q, Xia Y, Liu D. Comparison of procalcitonin and high-sensitivity C-reactive protein for the diagnosis of sepsis and septic shock in the oldest old patients. BMC Geriatr. 2017;17(1):173-3. 28764651.

36. Celik IH, Demirel FG, Uras N, et al. What are the cut-off levels for IL-6 and CRP in neonatal sepsis? J Clin Lab Anal. 2010;24(6):407-12. 21089127.

37. Ruan L, Chen GY, Liu Z, et al. The combination of procalcitonin and C-reactive protein or presepsin alone improves the accuracy of diagnosis of neonatal sepsis: a meta-analysis and systematic review. Crit Care. 2018;22(1):316. 30463590.

38. Yang Y, Xie J, Guo F, et al. Combination of C-reactive protein, procalcitonin and sepsis-related organ failure score for the diagnosis of sepsis in critical patients. Ann Intensive Care. 2016;6(1):51. 27287669.

\section{Figures}

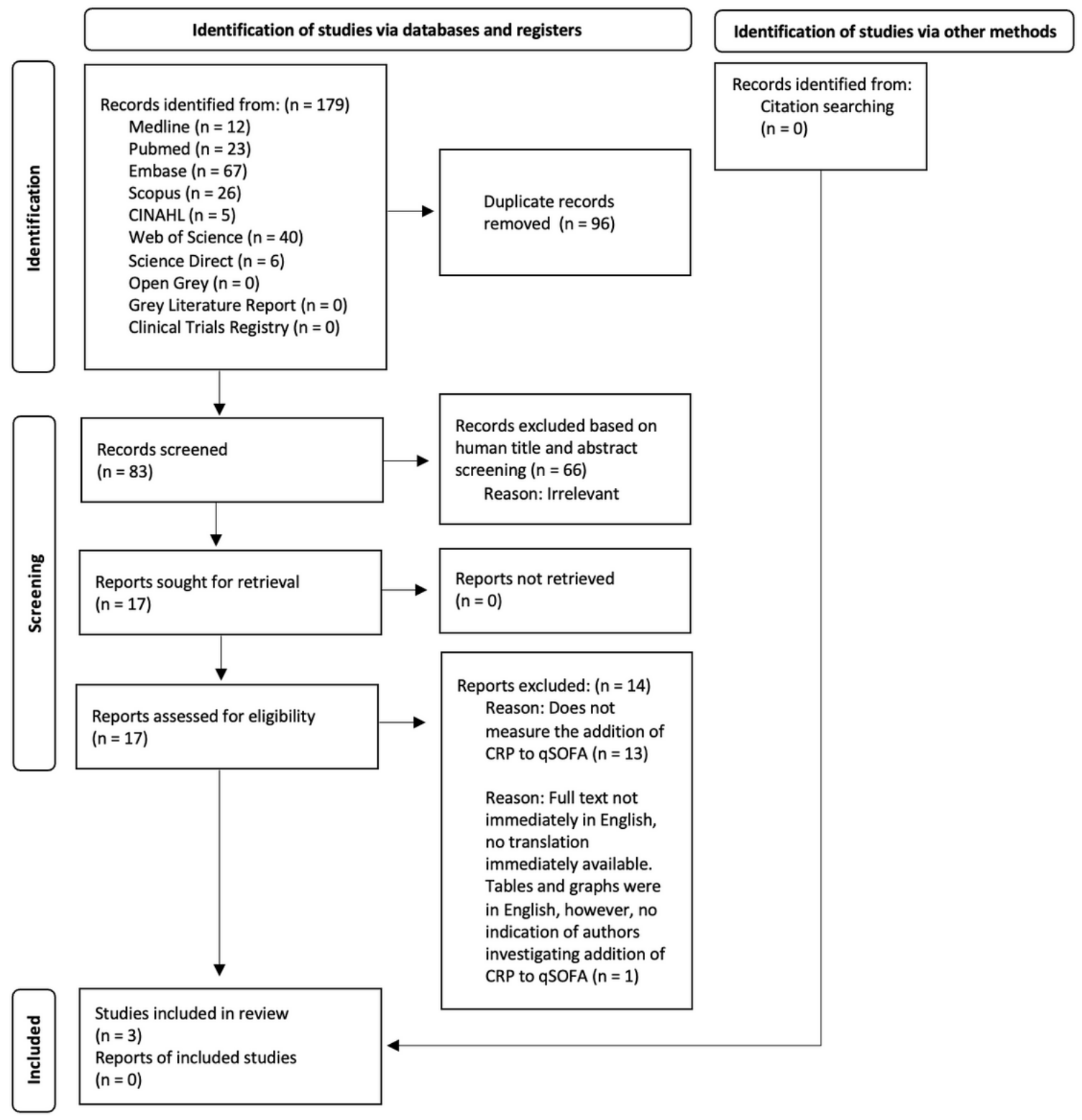

Figure 1

PRISMA flowchart 


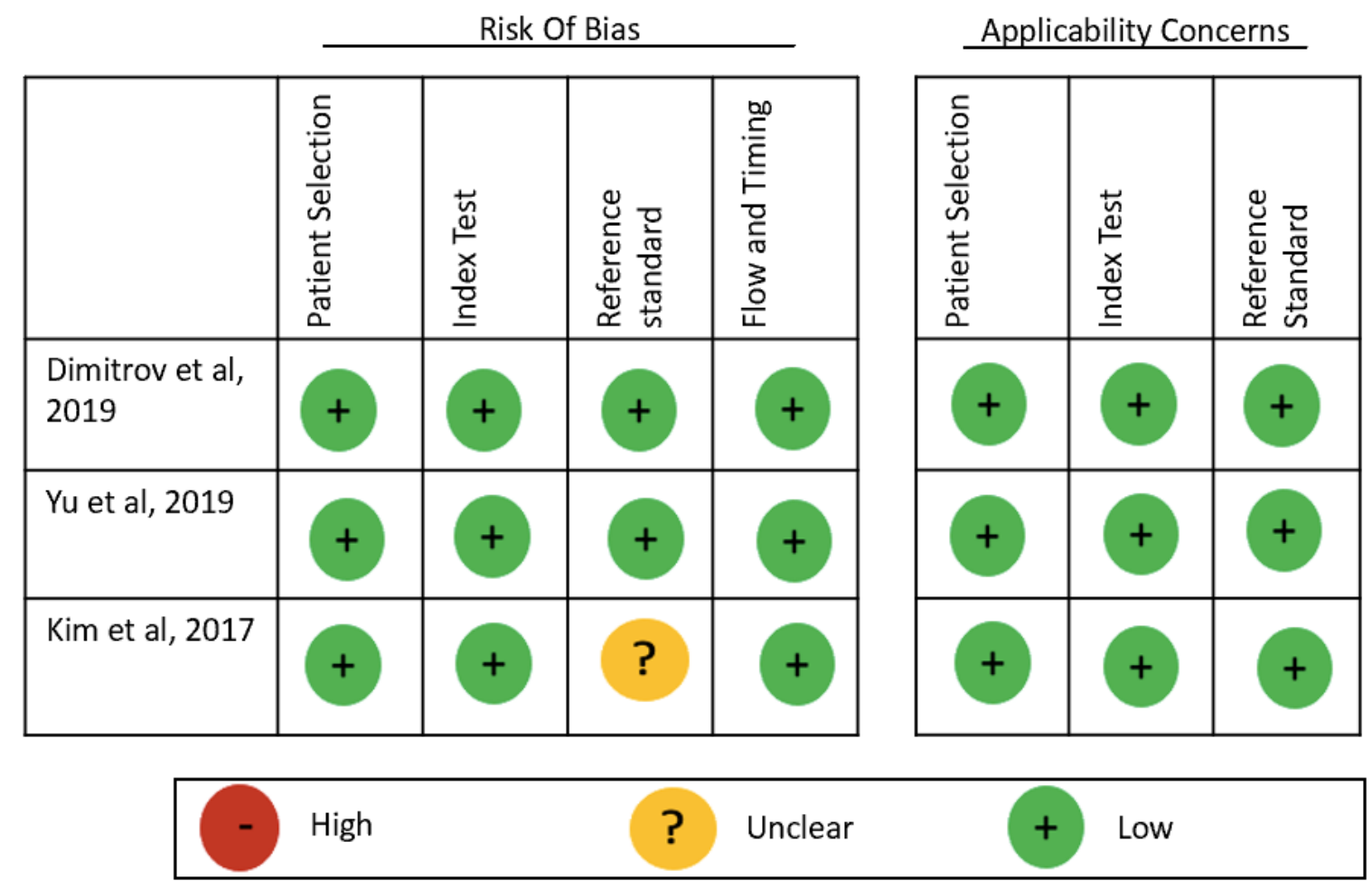

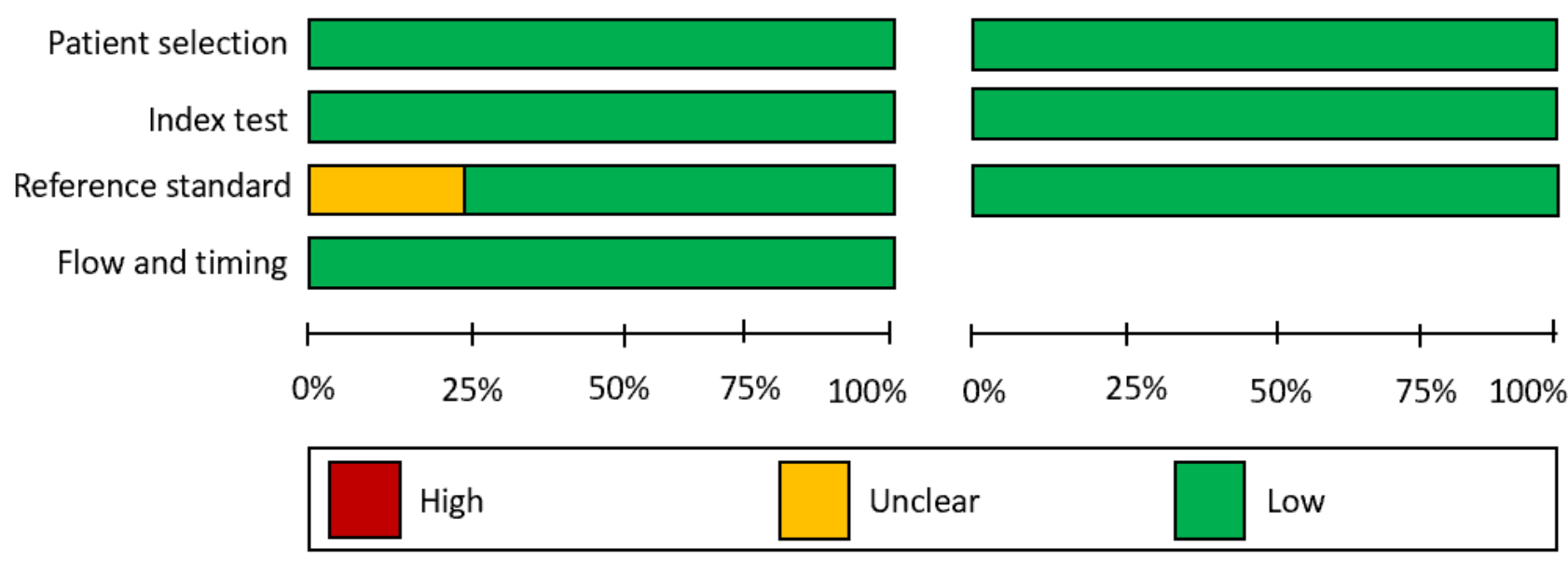

Figure 2

QUADAS-2 risk of bias summary of assessment

\section{Supplementary Files}

This is a list of supplementary files associated with this preprint. Click to download.

- Appendix.docx

- PRISMAchecklist.docx 